\title{
Desarrollo de un sistema inalámbrico escalable de medición de humedad del suelo en un cultivo de vid
}

\section{Development of a scalable wireless soil moisture measuring system for grape vine cultivation}

ORTEGA-CORRAL, César ${ }^{1} \dagger^{*}$, EATON-GONZÁLEZ, B. Ricardo ${ }^{2}$, LÓPEZ CRUZ, Florencio ${ }^{1}$, DÍAZSANTANA ROCHA, Laura Rocío ${ }^{1}$

${ }^{1}$ Universidad Tecnológica de Tijuana, Depto. de Tecnologías de la Información
${ }^{2}$ Universidad Tecnológica de Tijuana, Depto. de Tecnología Ambiental en Biotecnología

ID 1 ${ }^{\text {er }}$ Autor: César, Ortega-Corral / ORC ID: 0000-0001-5312-7521, CVU CONACYT ID: 90967 Candidato SNI

ID $1^{\text {er }}$ Coautor: B. Ricardo, Eaton-González / ORC ID: 0000-0003-4528-4740, CVU CONACYT ID: 94473

ID $2^{\text {do }}$ Coautor: Florencio, López Cruz / ORC ID: 0000-0002-8541-9569, CVU CONACYT ID: 295727

ID $3^{\text {er }}$ Coautor: Laura Rocío, Díaz-Santana Rocha / ORC ID: 0000-0002-8540-2595, CVU CONACYT ID: 467699

DOI: $10.35429 /$ JTEN.2020.14.4.20.30

Recibido 03 de Junio, 2020; Aceptado 30 Octubre, 2020

\section{Resumen}

Se presenta un sistema inalámbrico aplicado a la agricultura de precisión, se trata de nodos sensores que miden la humedad del suelo a diferentes profundidades en cultivos vitivinícolas, donde se aplica riego por goteo. La intención es implementar un sistema para facilitar el escalamiento, y crear una red de nodos (WSN, "Wireless Sensor Networks"), que se comuniquen por radiofrecuencia con una estación base (ET), para que se almacenen los datos localmente y que les de salida al Internet.

Sensores, Humedad del suelo, Red inalámbrica de sensores

\begin{abstract}
We present a wireless system applied to precision agriculture, made up of sensor nodes that measure soil moisture at different depths, applied to vine crops where drip irrigation is applied. The intention is to prepare a system for scaling, and to create a Wireless Sensor Network (WSN) that communicates by radio frequency with a base station (ET), so that the gathered data is stored locally and can be sent out an Internet gateway.
\end{abstract}

Sensors, Soil moisture, Wireless sensor networks

\footnotetext{
* Correspondencia del Autor (Correo electrónico: cesar.ortegac@uttijuana.edu.mx)

$\dagger$ Investigador contribuyendo como primer autor.
} 


\section{Introducción}

El agua dulce es un recurso que en muchas regiones cada vez es más escaso, y aunque en ciertos lugares se sigue practicando riego por inundación, en zonas de poca lluvia, donde no hay ríos, cuerpos de agua de almacenamiento y existen tierras que se consideran áridas y semiáridas, como en Baja California, el riego por goteo es necesario para optimizar el consumo hídrico (Medellín-Azuara et. al, 2013, Malinowski, 2004). En estos sitios, es entonces cuando la medición de la cantidad de humedad del suelo es imprescindible, dado que es útil para calcular la cantidad de agua que se requiere regar para mantener un balance hídrico favorable en el suelo para el tipo de cultivo, para el beneficio de la vegetación que crece y para no agotar los mantos freáticos (Bonamente et. al, 2015, Del Toro-Guerrero et al., 2018, Newhouse et. al., 2014).

La medición de humedad del suelo se suele hacer de diversos modos (Kaur et. al., 2016), tales como: 1) determinando el volumen de humedad en el suelo tomando una muestra de tierra, pesándola, luego se ingresan a un horno para secarla, y se vuelve a pesar, lo que constituye un método tardado y es tradicional; otra forma es 2) usando dispositivos mecánicos llamados "irriómetros" que mediante diferencias de presión determinando la tensión que produce el vapor de agua que contiene el suelo, en este método se requiere ir al sitio para ver el indicador de aguja, que muestra el contenido hídrico relativo; o la forma de mayor costo 3) usando instrumentación electrónica, para lo cual hay diversos sensores (resistivo-conductivos, capacitivos y de tiempo de reflexión electromagnética).

Tradicionalmente, los datos que se adquieren mediante este método, se guardan en un sistema tipo "data-logger", con la dificultad de tener que ir a campo por las mediciones, igual que al usar "irriómetros". Ahora, es posible usar tecnologías inalámbricas combinadas, como la creación de una red inalámbrica de sensores con el usó de alguna estación base, o de un "Gateway" que dé salida de los datos hacia el Internet por WiFi, o con el uso de módems GPRS ("General Packet Radio Systems) que permite transferencia de datos mediante telefonía celular.
Por lo que la tendencia en instrumentación a distancia es la telemetría, y ahora implementada aprovechando tecnologías del conocido "Internet of Things", o IoT, (Internet de las Cosas en español) (Shahzadi, 2016).

\section{Antecedentes \\ Humedad del Suelo}

La cantidad de humedad que puede retener el suelo de un cultivo tiene relación con de las cualidades de la tierra que lo constituye. Esto depende de los componentes o tipos del sustrato, tales como: arena, limo, o arcilla; usualmente es una combinación de algunos de éstos sustratos, como el suelo franco (o marga, que representa la combinación de arena, limo y arcilla en las mismas cantidades equivalentes). La arena es el sustrato que menos retiene agua, mientras que la arcilla tiene el mayor grado de retención hídrica.

La combinación de sustratos hace que los suelos tengan muy variadas proporciones de retención. La cantidad de humedad del suelo se puede medir de manera normalizada (volumen de agua por unidad de volumen de tierra, $\mathrm{cm}^{3} / \mathrm{cm}^{3}$ o $\mathrm{in}^{3} / \mathrm{in}^{3}$ ) o de forma relativa (por porcentaje de volumen de agua contra porcentaje de volumen de tierra). Ésta última es la forma preferida, pero no única, porque se puede aplicar a cualquier tipo de suelo, esto se ha estudiado extensamente y se han determinado rangos de humedad óptima para los cultivos de acuerdo al tipo de suelo.

En la Figura 1, se presenta una interpretación de dichos rangos, donde destacan tres regiones: 1) agua no disponible al margen de la "marchitez" (cuando la cantidad de agua es insuficiente para la vegetación), 2) agua disponible al margen de lo que se conoce como "capacidad de campo" (o cuando la cantidad del agua es suficiente y adecuada para la vegetación), y 3) saturación (o exceso de la cantidad de agua) por encima de la capacidad de campo, y es cuando la vegetación es afectada adversamente si se expone por tiempo prolongado a esta condición. Al estar saturado el suelo, la vegetación es incapaz de absorber agua y el efecto se invierte, el suelo entonces extrae el agua desde las raíces. 


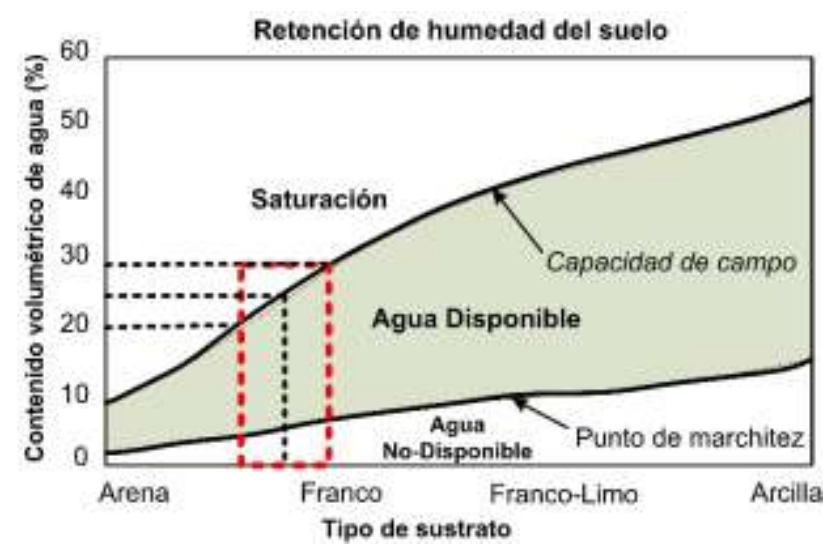

Figura 1 Rangos del contenido hídrico del suelo contra tipo de suelo

Fuente (Zotarelli et. al., 2010)

Cabe mencionar que en la Figura 1, el área marcada por el recuadro con acotamiento rojo, indica el tipo de suelo característico de la zona del Valle de Guadalupe, B.C., se trata de tierra mezclada entre tierra franca y arena caliza. Lo que implica rangos de humedad de: (1) marchitez debajo de $7 \%$ en promedio, (2) agua disponible de $8 \%$ a $24 \%$, al límite de la Capacidad de Campo, y (3) saturación del suelo de $25 \%$ en adelante. Entre más cerca al cauce de algún arroyo o río el sustrato es más arenoso.

Hay diferentes criterios entre agricultores sobre el manejo de humedad del suelo, uno de los más aceptados es que se active y desactive el riego para mantener los niveles por encima de la marchitez y por debajo de la capacidad de campo. Pero este criterio depende de la disponibilidad inmediata del recurso hídrico para efectuar el riego, lo cual depende del costo del agua y del presupuesto disponible. Otro criterio, particularmente en viñedos con recursos hídricos limitados, y que se aplica por cuestiones presupuestales justo semanas antes de la cosecha, es el de efectuar el riego por encima de la capacidad de campo y mantener saturada la tierra en dos o tres días para que el fruto aumente de volumen lo más posible sin dañar las raíces de la parra, y se hace en ráfagas supervisadas a partir de que se tenga suficiente agua. Con base en lo anterior y de acuerdo a la práctica agrícola de riego en el sitio de estudio, en este trabajo, se puso a prueba el prototipo para la medición de humedad del suelo que aquí se presenta, justo en un viñedo dónde se aplica dicho criterio de saturación corta antes de la cosecha, y los resultados que se obtuvieron reflejan la variabilidad hídrica de acuerdo a tales acciones de riego.

\section{Sensores de humedad del suelo}

Los sensores disponibles para medir la humedad del suelo tienen en común un tiempo de vida relativamente corto, de semanas o días. Los sensores resistivos-conductivos son los más frágiles, deben estar en contacto directo con tierra húmeda y sus láminas conductivas se corroen o sulfatan en pocos días bajo esas condiciones (Brillante et. al, 2015). Entre más húmedo o conductivo está el sustrato del suelo, en menor tiempo se dañan los puntos de contacto por corrosión.

A estos sensores le siguen los del tipo capacitivo (Radi et. al. 2018), usualmente compuestos también por dos placas cuyo dieléctrico es el sustrato donde se entierran, y aunque suelen tener un recubrimiento impermeable, éste es muy delgado con la intensión de no afectar su sensibilidad ante cambios de capacitancia del suelo; y en consecuencia, los sensores capacitivos al estar por tiempo prolongado enterrados en un suelo húmedo, tal recubrimiento se daña hasta que permite que la humedad se ponga en contacto con las placas metálicas en su interior, y dónde se acumula agua hasta que fallan después de unas semanas.

Por último, los sensores más longevos son los sensores reflecto-métricos del dominio del tiempo (TDR, "time domain reflectometric"), operan bajo el principio de reflexión, están compuestos por dos electrodos de acero inoxidable o placas conductivas aisladas, desde uno de los electrodos se emite una señal EM que refleja el electrodo vecino (Dalton, 1986); su circuitería electrónica determina el tiempo que tarda en reflejarse la señal EM el cual es proporcional a la humedad que hay en el sustrato donde está enterrado el sensor, por lo que es un dispositivo complejo y de alto costo, esto impide que se pueda escalar dicha tecnología por su alto costo. La fragilidad del sensor TDR reside en su electrónica, que se daña fácilmente en presencia de humedad, pero su tiempo de vida se prolonga meses o quizá años comparándolo con sensores de bajo costo, lo cual depende de la calidad de su fabricación. En este trabajo, se usaron sensores del tipo capacitivo de humedad del suelo, o "capacitive soil moisture" SKU:SEN0193, Figura 2, porque son más duraderos que los conductivos, y de mucho menor costo que los TDR. 


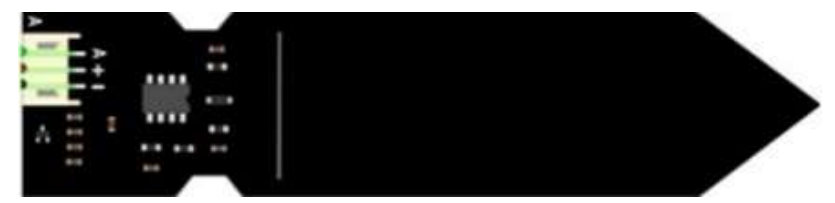

Figura 2 Sensor capacitivo SKU:SEN0193 que mide la capacitancia del suelo, y de ahí se infiere el contenido de humedad

Por lo regular, los sensores capacitivos, como el de la Figura 2, los venden con la electrónica expuesta, por lo que hay que agregarles un recubrimiento impermeable. Así mismo, la parte debajo de la línea blanca del sensor SKU:SEN0193 es el capacitor, formado por dos placas que están ligeramente cubiertas con pintura, para que afecte a su sensibilidad lo menos posible.

Por otra parte, este tipo de sensor (al igual que el tipo conductivo) se usa mayormente como un indicador discreto de tres estados de humedad: (1) tierra seca, (2) tierra húmeda y (3) tierra saturada con agua. En este trabajo, se decidió implementar el sensor capacitivo SKU:SEN0193 (o simplemente SEN0193) usando una referencia calibrada para hacer la comparación en inferencia a partir de su respuesta.

\section{Tierra de cultivo y calibración de sensores de humedad del suelo}

En el proceso de caracterizar la respuesta de los sensores capacitivos de humedad del suelo, se utilizó como referencia un sensor de alto valor, conectado a un sistema de acondicionamiento y de interfaz con el usuario llamado LabQuest, Figura 3, de la marca Vernier (Vernier Software and Technology, 2017).

Esté dispositivo con su sensor de humedad del suelo, arroja valores de humedad relativa del suelo de $0 \%$ a $100 \%$. Y optamos por calibrar la referencia a partir de muestras de tierra de un sitio llamado Viñedo El Mogor, ubicado en el Valle de Guadalupe, Baja California, que es donde finalmente se hicieron las pruebas de campo.
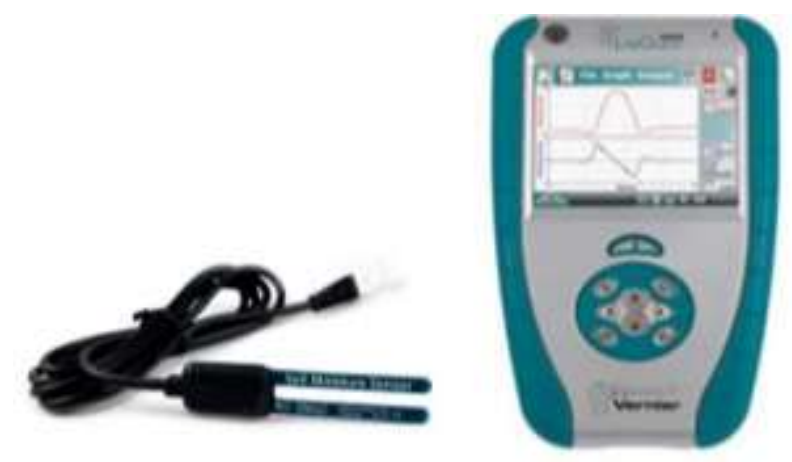

Figura 3 Sensor de humedad del suelo y plataforma LabQuest usados como referencia para calibrar sensores Fuente: Elaboración propia

El procedimiento para la calibración fue a partir de muestras de suelo extraídas del Valle de Guadalupe, las muestras se secaron en un desecador, y se agregó un volumen medido de agua, tal que en el rango inferior fue una mezcla de $95 \%$ de tierra y $5 \%$ de agua, y el rango superior fue una mezcla de $70 \%$ de tierra y $30 \%$ de agua (que adquirió la textura de lodo).

\section{AgroNodo: Nodo inalámbrico de sensores de humedad del suelo}

En este desarrollo, se integró un nodo inalámbrico con cuatro sensores capacitivos de humedad del suelo SEN0193, incluyendo un sensor de temperatura y humedad relativa del aire SHT-10, con protector resistente a la intemperie colocado dentro de un escudo solar. El sistema microcontrolador es el conocido Arduino MEGA, se seleccionó por facilidad de una rápida implementación y que tiene varios puertos serie y varias entradas analógicas, para futura expansión (Arduino, 2020). En cuanto a los sensores capacitivos SEN0193, son de muy bajo costo, sus placas tienen un recubrimiento impermeable delgado color negro, su electrónica la constituye un LM555 (Texas Instruments Inc., 2015), el cual produce una señal periódica cuadrada de voltaje que cambia con la proporción de humedad en el suelo donde se entierran las placas.

El nodo es inalámbrico porque se enlaza con una estación base mediante radios XBee Pro operando a $2.4 \mathrm{GHz}$, con alcance en distancia de hasta $1.5 \mathrm{Km}$ (Digi International, 2009). Con el protocolo DigiMesh, se crean redes de XBee, ampliando el alcance en la comunicación de datos (Digi Intl., 2016). En la Figura 4 se muestra la integración de los componentes del nodo de sensores, que aquí se denomina AgroNodo. 


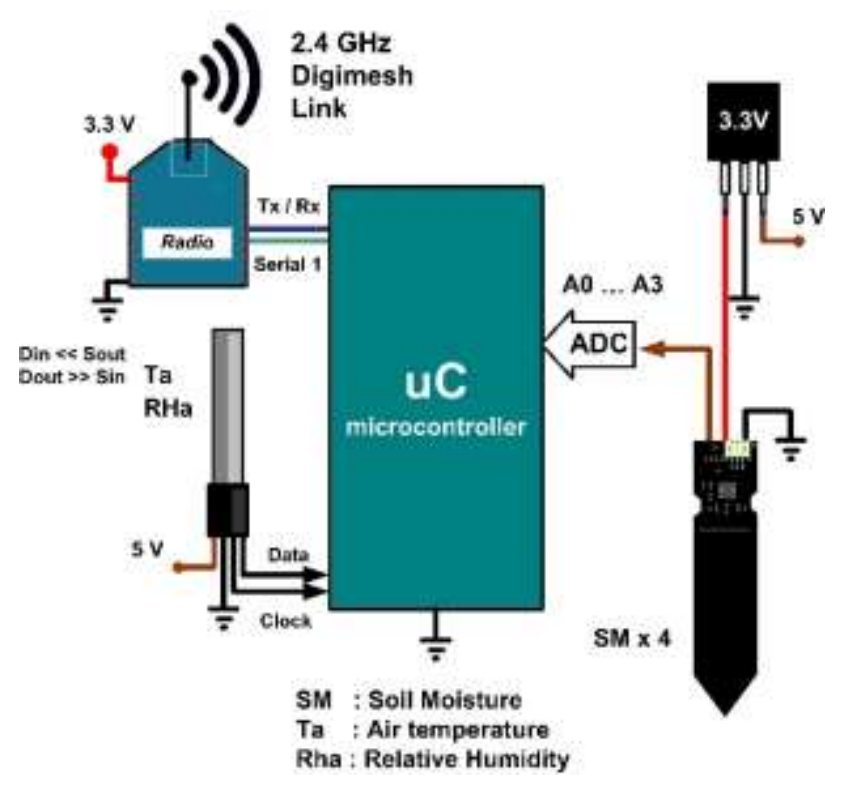

Figura 4 Integración del nodo de sensores inalámbrico Fuente: Elaboración Propia

Los cuatro sensores SKU:SEN0193 arrojan valores útiles para determinar el grado de penetración del agua en el suelo de cultivo; así como de la retención hídrica del sustrato particular. Los sensores se colocan a diferentes profundidades de tierra, cercanas a las raíces de la vegetación que se cultiva, en este caso en las raíces de la parra de interés.

Por otro lado, para que el AgroNodo opere en el campo, lejos de conexiones eléctricas, se alimenta con una batería de $12 \mathrm{~V}$ de $\mathrm{CD}$ de 7Ah (Amper-horas) de capacidad. Esta batería se recarga a través de un controlador de carga de $12 \mathrm{~V}$ alimentado por un panel fotovoltaico poli-cristalino de $17 \mathrm{~V}$ CD y de hasta $50 \mathrm{~W}$.

\section{AgroBase - Estación Base de la red de sensores de humedad del suelo}

La tarea de los nodos de sensores inalámbricos, es de recolectar datos de humedad del suelo y medio ambiente, y crear un mensaje con un protocolo y de transmitirlo periódicamente, dirigido a un destinatario particular, usualmente llamado estación base (o BS, "Base station") (More, 2016).

En este caso, en el centro de la BS se usa la computadora miniatura conocida como Raspberry PI 3 Model B+, o RPI, con sistema operativo conocido como Raspbian (Raspberry PI, 2020).
EL RPI funciona como servidor ligero y puesto en interfaz con un radio digital XBee Pro, habilitado el protocolo DigiMesh para asegurar la entrega de paquetes, a través de software desarrollado en Python, se crean redes inalámbricas que transmiten mensajes codificados con los datos de los sensores de humedad del suelo, temperatura y humedad del aire. La estación base se muestra en la Figura 5.

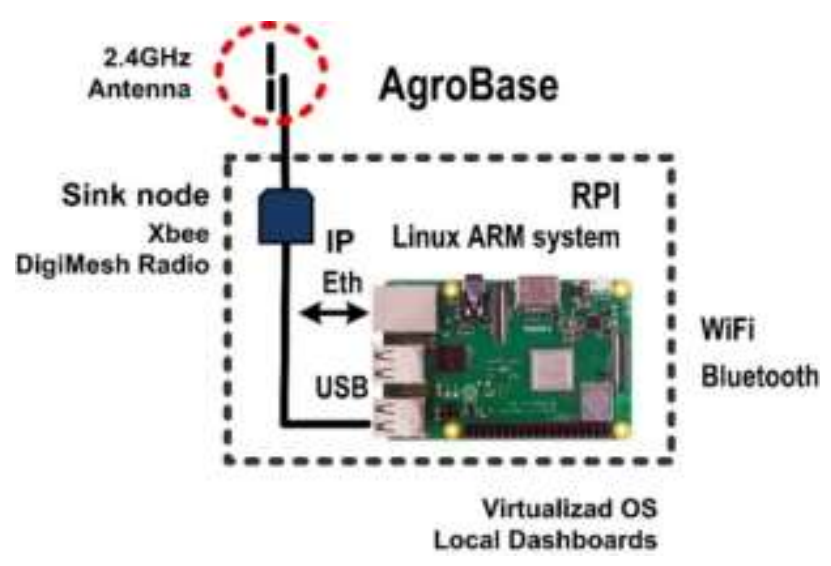

Figura 5 Componentes de la Estación Base. Fuente: Elaboración Propia

El módulo XBee Pro habilitado con DigiMesh, protocolo propietario del fabricante, es capaz de crear redes en malla, estableciendo las rutas más confiables para garantizar entrega inalámbrica de datos. En la Figura 6 se muestra la topología de un sistema completo de riego por goteo usando esta WSN, que incluye la posibilidad de controlar bitácoras de riego a distancia, con un nodo especializado en abrir o cerrar una electroválvula del sistema de mangueras de riego por goteo.

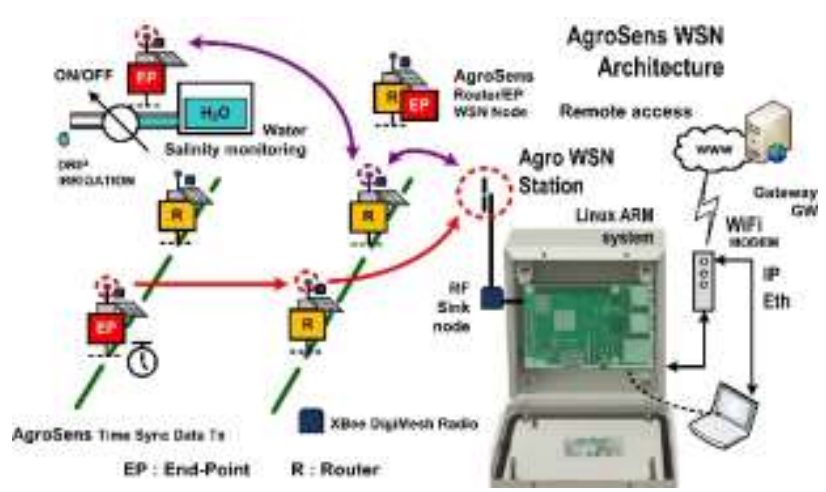

Figura 6 WSN de multi-saltos con salida al gateway Fuente: Elaboración Propia

En la Figura 6, los nodos sensores terminales o "end points", EP, son nodos que generan mensajes que contienen datos adquiridos periódicamente de los sensores y que se destinan a la estación base, que aquí es llamada Agro WSN Station. 
Los nodos cuando no son EP, son enrutadores o "routers", R, sirven de puntos multi-salto en la red de nodos que se crea para asegurar que los mensajes logren su destino. En la Figura 7 se muestra el diagrama a bloques del algoritmo de muestreo que se ejecuta en el nodo sensor.

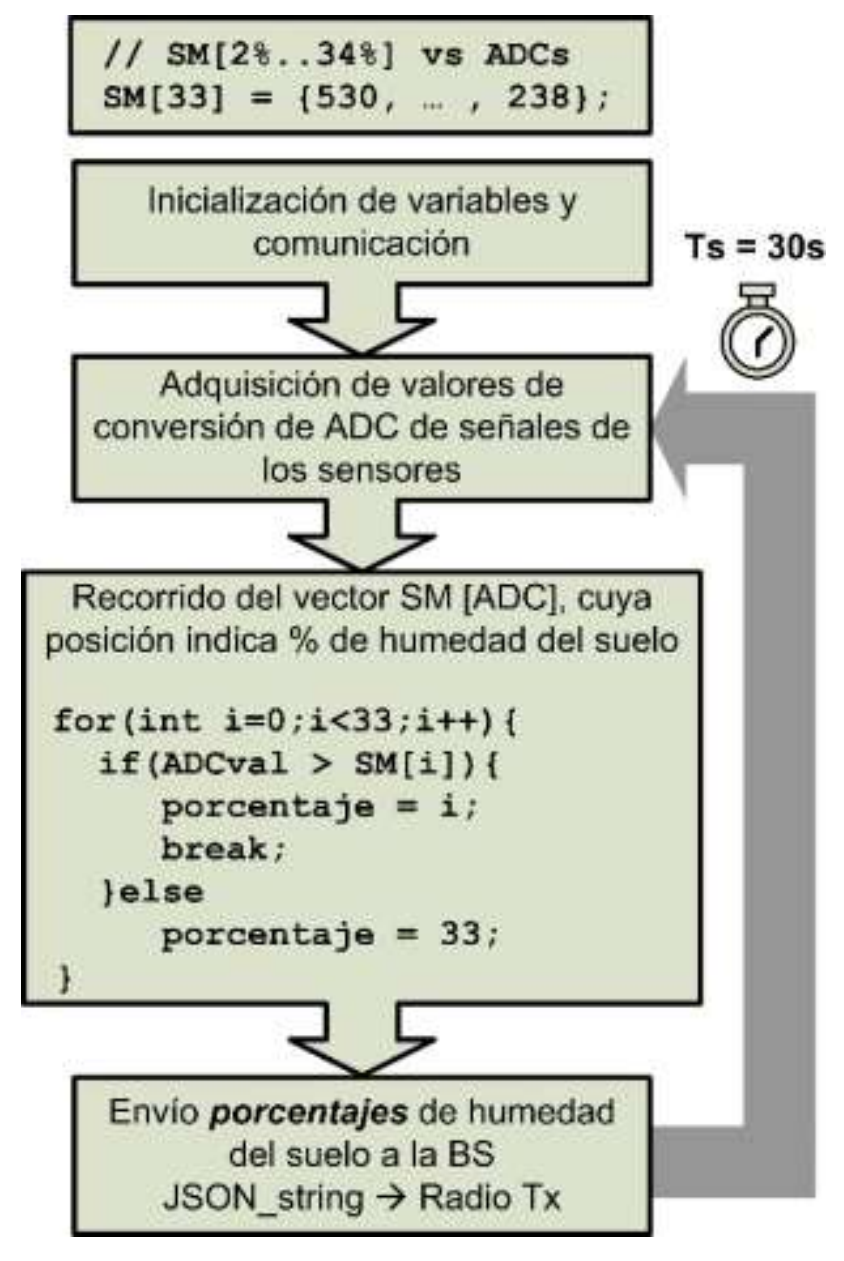

Figura 7 Diagrama a bloques del algoritmo del nodo Fuente: Elaboración Propia

En el momento que se cumple el periodo de muestreo y de envío, los nodos sensores se vuelven EP. Los mensajes de los Agro-Nodos se codifican usando el conocido protocolo JSON ("JavaScript Object Notation"), mediante el cual se pueden estructurar los datos para facilitar su extracción del mensaje. Por otro lado, para evitar hacer cálculos después del muestreo, el software de los nodos sensores SEN0193 incorporan un vector denotado por SM[ ], el cual representa un arreglo de valores de conversión analógica a digital (dentro de un rango de 10 bits) contra valores medidos con la referencia calibrada Vernier LabQuest. Es decir, ya se había hecho la caracterización de los sensores SEN0193 a diferentes contenidos de humedad, usando muestras preparadas de tierra extraída del campo.
Y como se mencionó, este método se implementa porque no se requieren cálculos en el microcontrolador, lo cual optimiza el uso de recursos de sistema digital. Los valores del vector SM del algoritmo del AgroNodo, de acuerdo al tipo de sustrato obtenido, se encuentran entre 530 cuentas del ADC para $2 \%$ de humedad, y desciende hasta 238 cuentas del ADC cuando se mide $33 \%$ de humedad por el LabQuest. El sustrato particular con el que se trabaja se satura en alrededor $30 \%$.

Del otro extremo, el programa de la estación base, se desarrolló usando el lenguaje conocido como Pyhton, es nativo de los sistemas operativos Linux, y en Raspbian del RPI no es excepción. El radio XBee con antena de $2 \mathrm{~dB}$ se instaló en un módulo de interfaz USB, y se conectó a uno de los puertos del RPI. Mediante Python se ejecutan las instrucciones que leen los datos que recibe del XBee mediante USB, en este caso de los nodos de la misma WSN.

La estación base envía cada 30 segundos un mensaje de sincronía en un esquema TDM ("Time División Multiplexing"), bajo el cual cada nodo en la red envía su mensaje de mediciones en el turno que le corresponde para evitar colisión de envíos conforme crezca la WSN. Por otro lado, cuando la BS recibe un mensaje de algún AgroNodo, éste pasa por un proceso de decodificación de acuerdo al protocolo JSON. En la Figura 8 se muestra el diagrama a bloques del software que se ejecuta en la Estación Base. 


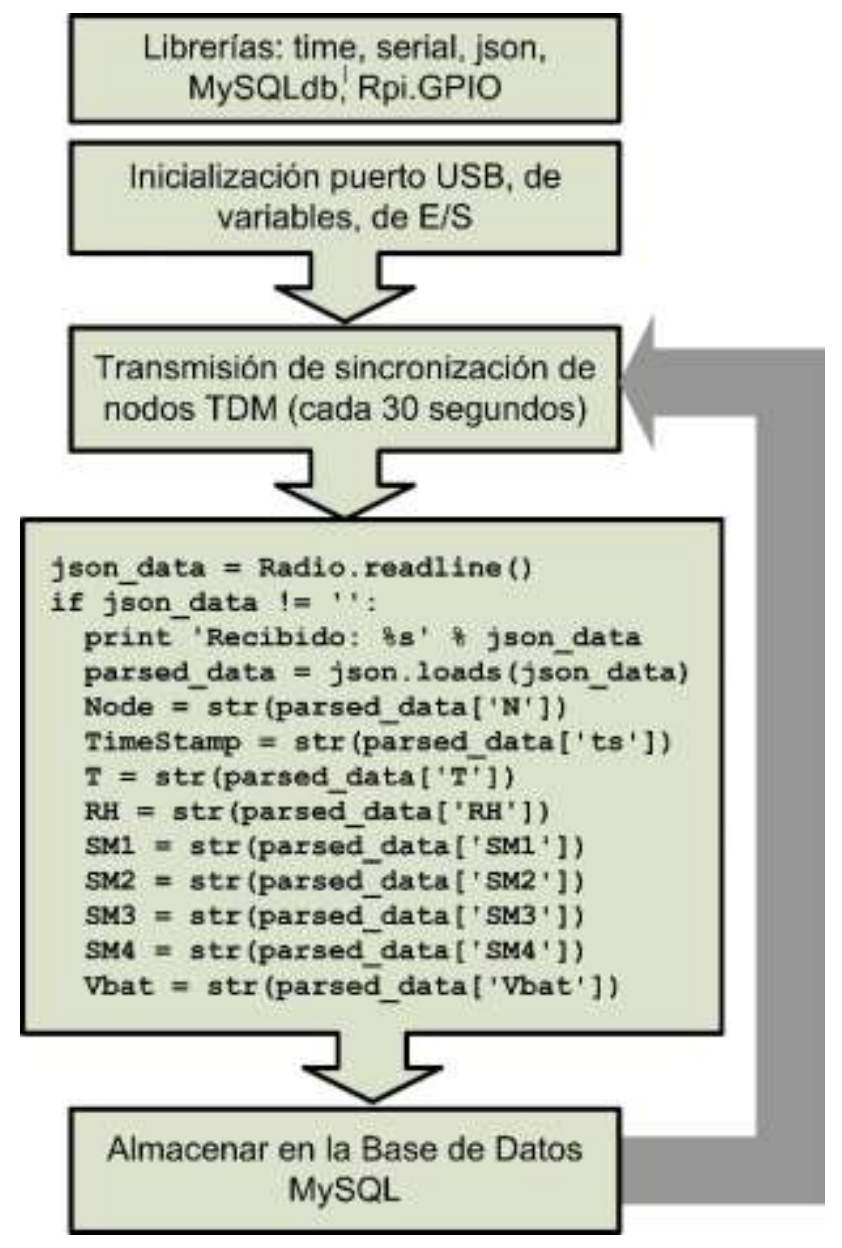

Figura 8 Diagrama a bloques del algoritmo la Estación Base

Fuente: Elaboración Propia

Después que el software de la Estación Base logra decodificar el mensaje JSON que proviene de un AgroNodo, procede extrayendo los datos que contiene. Luego la BS realiza una conexión a su Base de Datos MySQL y procede en almacenar los datos respectivos en la tabla correspondiente del AgroNodo.

\section{Pruebas iniciales de campo}

Para determinar el desempeño real, se instaló una estación base y un AgroNodo en el Viñedo El Mogor, del Valle de Guadalupe, Baja California. El nodo se colocó justo debajo de una parra, y los sensores SEN0193 se enterraron al costado de las raíces, a cuatro profundidades (10, 20, 30 y $40 \mathrm{~cm}$ ) Figura 9.

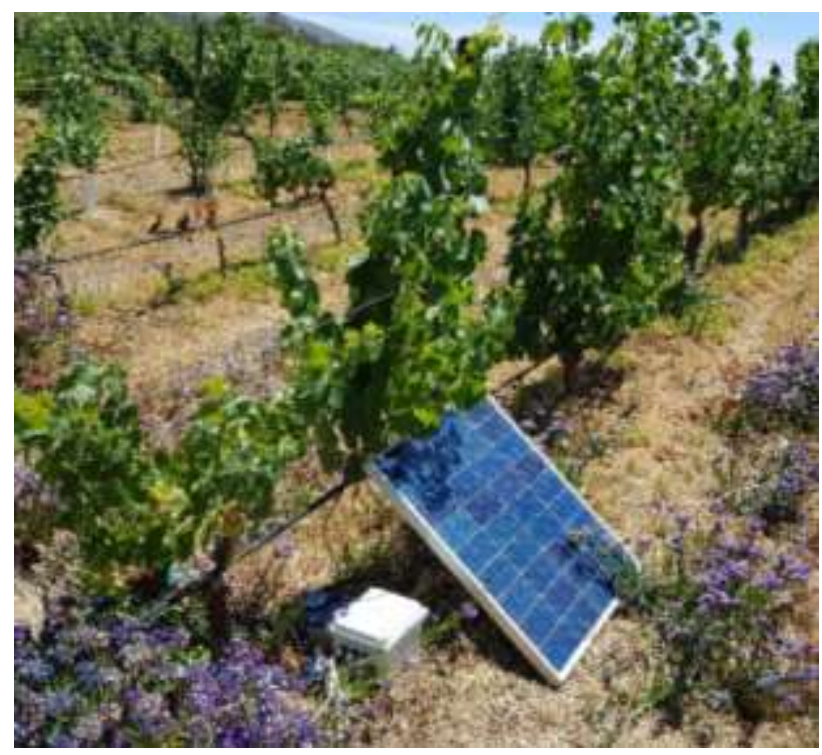

Figura 9 Nodo de sensores con su respectivo panel solar Fuente: Elaboración Propia

Las mediciones iniciales usando los sensores SEN0193 registraron valores inestables desde saturación hasta en cero, como se observa en la Figura 10; lo cual dio una indicación que los sensores SEN0193 fueron afectados adversamente por la humedad.

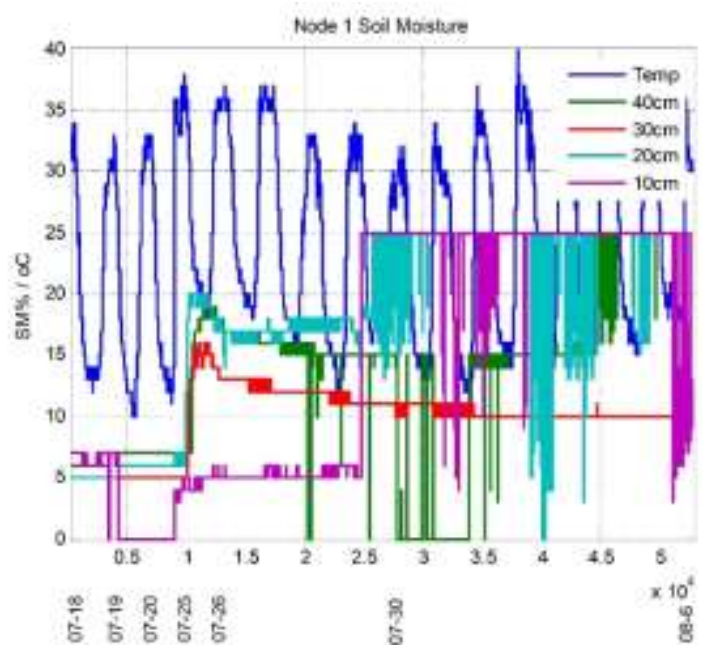

Figura 10 Mediciones de temperatura y de humedad del suelo a cuatro profundidades, se dañaron tres sensores de humedad del suelo

Fuente: Elaboración Propia

Después de inspeccionar a los sensores SEN0193 retirados del suelo, fue visible el daño de los sensores debido a que el suelo se saturó con un goteo de 36 horas, y aparentemente el aislante de los sensores fue insuficiente para evitar las fallas. El sensor SEN0193 superficial a $10 \mathrm{~cm}$ de profundidad se dañó desde su instalación, posiblemente porque se raspó y se le cayó el aislante. 
En las profundidades de 20, 30 y $40 \mathrm{~cm}$, respectivamente, los sensores funcionaron correctamente a partir de su instalación y desde el inicio del riego por goteo; pero después del segundo día del goteo, el sensor de $40 \mathrm{~cm}$ de profundidad registró lecturas fallidas, seguido dos días después por el sensor a $20 \mathrm{~cm}$. En cambio, el sensor SEN0193 a $30 \mathrm{~cm}$ de profundidad funcionó y registró valores estables hasta el momento de su extracción. En la Figura 11 se muestran los sensores SEN0193 dañados $(10 \mathrm{~cm}, 20 \mathrm{~cm}$ y $40 \mathrm{~cm})$.

En el primer caso, $10 \mathrm{~cm}$, al instalar el sensor se removió la pintura aislante poniendo en contacto directo a la placa de cobre; es decir, se raspó el sensor con el sustrato. Por otro lado, los otros sensores SEN0193 se dañaron debido a que la tierra saturada de humedad, y el peso de la misma sobre el sensor debilitó al recubrimiento aislante y provoco ingreso de humedad mojando sus placas de cobre, y mitigando la medición capacitiva.

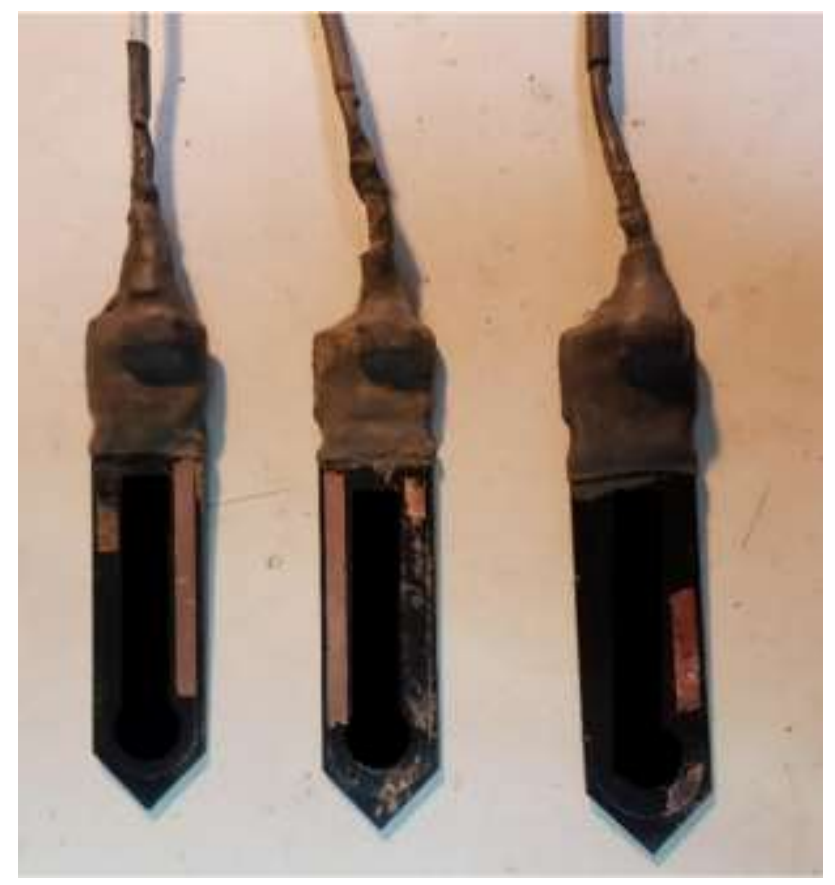

Figura 11 Sensores SEN0193 dañados, la humedad permeó su recubrimiento de pintura

Fuente: Elaboración Propia

Para resolver el problema de daño de los sensores SEN0193, se buscaron alternativas para recubrir a los sensores con capas adicionales de aislante, lo cual afectó adversamente a su sensibilidad en las mediciones, se eliminó por completo el recubrimiento se pintó de nuevo con pintura vinílica, lo cual también empeoró su sensibilidad.
Lo que funcionó fue usar un recubrimiento muy ligero de hule líquido, Figura 12, el cual se deposita con aerosol, se agregó en capas muy delgadas, reduciendo la sensibilidad en el menor grado posible, su respuesta fue verificada comparándola con la referencia LabQuest.

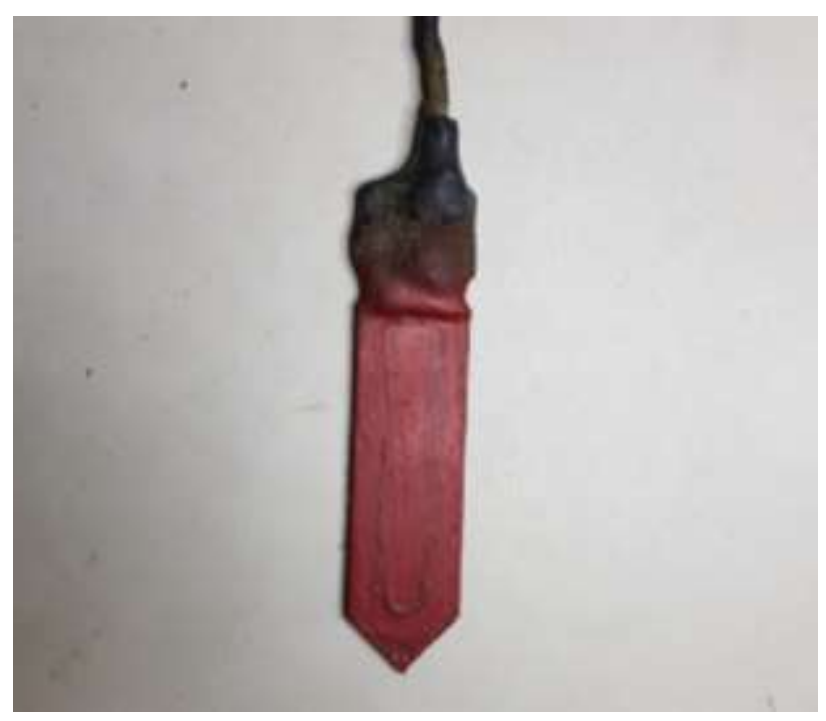

Figura 12 Sensor CSM cubierto por hule Fuente: Elaboración Propia

Se procedió en re-calibrar usando tierra preparada previamente con diferentes contenidos de humedad. Esto permitió volver a obtener puntos discretos de las cuentas del ADC contra porcentajes de humedad medidos de cada muestra con el Vernier LabQuest, aunque el rango se redujo, fue posible precisar valores discretos en pasos de $1 \%$ cercano a la zona de saturación (de $25 \%$ a $33 \%$ ). Con estos ajustes hechos en el software del AgroNodo, se realizó una segunda implementación de los sensores SEN0193 y fueron puestos en operación durante una semana, para obtener gráficas históricas de la humedad a diferentes profundidades del suelo, y determinar el grado de afectación que tiene el proceso de riego por goteo a lo largo de días consecutivos.

\section{Resultados}

La re-instalación de los sensores fue en el mismo sitio, Viñedos El Mogor, y aunque la sensibilidad de los sensores se redujo por el recubrimiento de hule, fue aceptable la variación de $+/-1 \%$ con respecto a la escala completa. En las Figura 13 a la Figura 16 se muestran los valores de humedad del suelo de ocho días; dónde se logró medir de manera remota el riego por goteo, a diferentes profundidades: 
El riego se activó durante tres horas en el 3er día, también en el 4to día y lo mismo en el 5to día de la implementación. La intención del administrador del campo de cultivo fue saturar el suelo días antes de la cosecha para que el fruto se hidratara al máximo

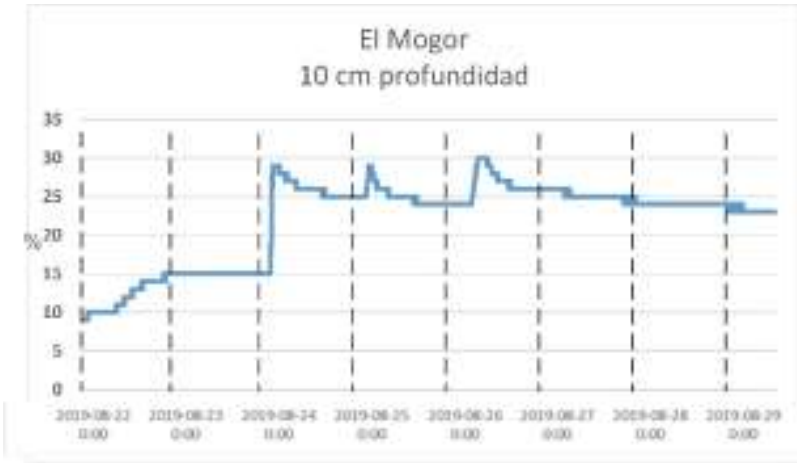

Figura 13. Humedad del suelo a $10 \mathrm{~cm}$ de profundidad Fuente: Elaboración Propia

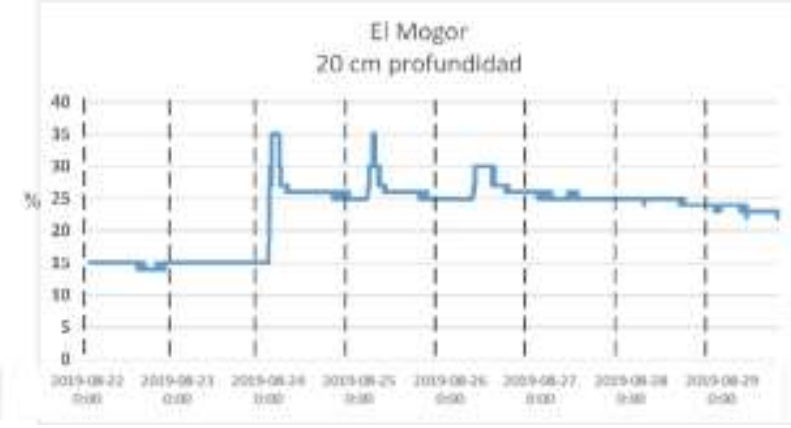

Figura 14. Humedad del suelo a $20 \mathrm{~cm}$ de profundidad Fuente: Elaboración Propia

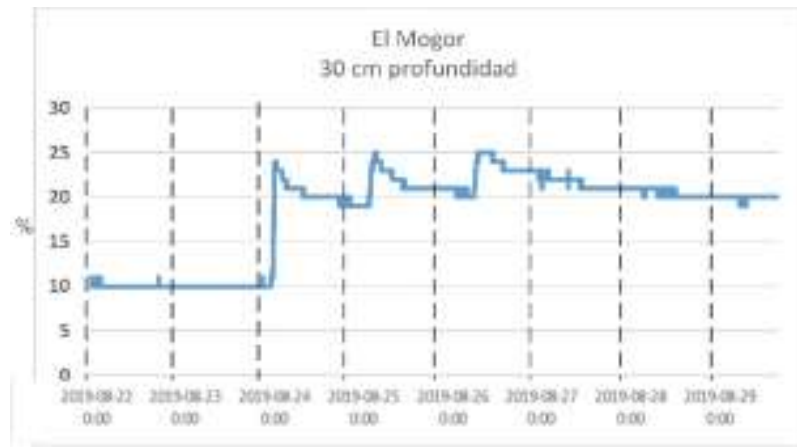

Figura 15. Humedad del suelo a $30 \mathrm{~cm}$ de profundidad Fuente: Elaboración Propia

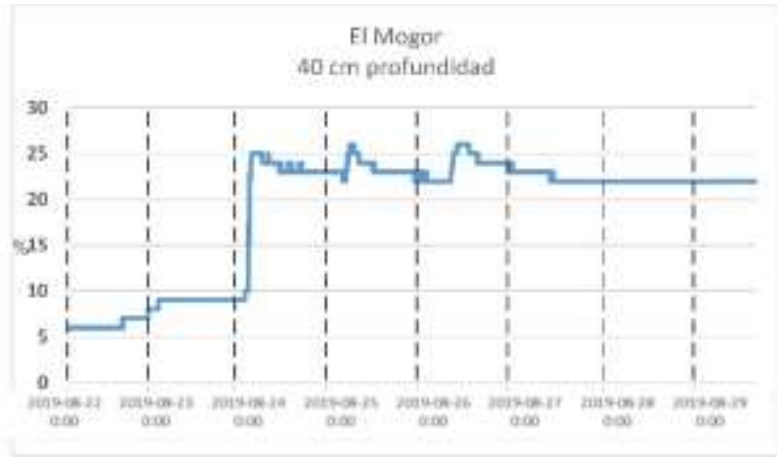

Figura 16 Humedad del suelo a $40 \mathrm{~cm}$ de profundidad Fuente: Elaboración Propia
Según las figuras 13 a la 16, la retención de humedad fue menor a $10 \mathrm{~cm}$ de profundidad, se infiere que la radiación solar estimula la evaporación por ser una región cercana a la superficie. Y en general se observa que la humedad que se filtró se retiene en buena medida a otras profundidades, pues sólo bajó $5 \%$ por día a $30 \mathrm{~cm}$ y $40 \mathrm{~cm}$ de profundidad, y se mantuvo así en el margen entre saturación y de capacidad de campo.

\section{Agradecimiento}

Agradecemos a la Gerencia de Viñedos El Mogor, del Valle de Guadalupe, B.C., México, por su disposición en apoyar a todo tipo de experimentación científica en sus instalaciones. $\mathrm{Y}$ agradecemos particularmente al Ing. Santiago Torres, agrónomo que administra los campos de cultivo de El Mogor. El Ing. Torres nos permitió acceso para realizar la instalación de la estación base $\mathrm{y}$ nodo de sensores, y en visitas subsecuentes. Fue valiosa su participación también porque sugirió en qué punto del viñedo sería adecuado instalar los sensores para su mejor apreciación del riego por goteo.

\section{Conclusiones}

Fue posible la medición de la humedad del suelo de manera remota y a través de la red de nodos establecida, se concluye que la implementación del sistema es posible y es escalable, también que el sistema puede mantenerse operando indefinidamente por el uso de energía solar para mantener a los nodos funcionando, situación altamente favorable en sitios como los viñedos en el Valle de Guadalupe, dado que comúnmente no se cuenta con suministro de energía eléctrica en campo o cercana a los sitios de cultivo.

La limitante en implementar este tipo de sistemas son propiamente los sensores de humedad del suelo, ya que son afectados por las condiciones físicas adversas al estar enterrados en tierra húmeda y saturada, además de que el tipo de suelo por su textura y contenido de sal, pueden extremar las micro-condiciones de operación del sensor. Los sensores capacitivos de bajo costo no están hechos para operación prolongada bajo tales condiciones. El recubrirlos con capas adicionales de aislante de humedad mitiga su sensibilidad y reduce el rango de las mediciones. 
Por lo que debe hacerse un análisis cuidadoso del costo beneficio que implica el uso de dichos sensores, pues si se tienen que sustituir continuamente, la ganancia de implementar tales sensores se reduce o anula por el costo operativo de hacer trabajo de campo para tales efectos, en lugar de colocar sensores longevos, como los TDR, aunque implique elevar el costo del sistema.

\section{Referencias}

Arduino (2020). Arduino MEGA 2560 https://store.arduino.cc/usa/mega-2560-r3.

Brillante, L., Mathieu, O., Bois1, B., Van Leeuwen, c, \& Lévêque, J.. (2015). The use of soil electrical resistivity to monitor plant and soil water relationships in vineyards. SOIL, 1, 273286, 2015. www.soil-journal.net/1/273/2015/. doi:10.5194/soil-1-273-2015.

Bonamente, E., Scrucca, F., Asdrubali, F., Cotana, F. \& Presciutti, A.. (2015). The Water Footprint of the Wine Industry: Implementation of an Assessment Methodology and Application to a Case Study. Sustainability Open Access Journal. 12190-12208;

Bongiovanni, R., Lowenberg-DeBoer, J. (2004). Precision Agriculture and Sustainability. Journal Precision Agriculture 5(4):359-387. DOI: 10.1023/B:PRAG.0000040806.39604.aa

Dalton, F.N., Van Genuchten, M.Th.. (1986). The time-domain reflectometry method for measuring soil water content and salinity. Elsevier Pub. Geoderma Journal. Volume 38, Issues 1-4, September 1986, Pages 237-250.

Del Toro-Guerrero, F.J., Vivoni, E.R., Kretzschmar, T, Runquist, S.H.B., \& VázquezGonzález, R.. (2018). Variations in Soil Water Content, Infiltration and Potential Recharge at Three Sites in a Mediterranean Mountainous Region of Baja California, Mexico. Water, Open Access Journal (ISSN 2073-4441).

Digi International Inc. (2009). XBee®/XBeePRO® RF Modules. Product Manual v1.xEx 802.15.4 Protocol.

Digi Intl. (2016). XBee/XBee-PRO DigiMesh 2.4. Radio Frequency (RF) Module User's Guide.
Kaur, K., Mahajan, R., Bagai, D. (2016). A Review of Various Soil Moisture Measurement Techniques. International Journal of Innovative Research in Science, Engineering and Technology. ISSN (Online): 2319-8753. ISSN (Print): 2347-6710

Mackenzie, D.E., Christy, A.G.. (2005). The role of soil chemistry in wine grape quality and sustainable soil management in vineyards. Water Science and Technology. Vol. 51, No 1 pp 2737.

Malinowski, J.P. (2004). Water Supply and Prospects in Baja California. Master's Thesis. University of California at Davis. U.S.A.

Medellín-Azuara, J., Mendoza-Espinosa, L., Pells, C., \& Lund, J.R.. (2013). Pre-Feasibility Assessment of a Water Fund for the Ensenada Region Infrastructure and Stakeholder Analyses. The Nature Conservancy.

More, S.N., Nighot, M. (2016). A Review of Wireless Sensor Network for Agriculture. International Journal on Recent and Innovation Trends in Computing and Communication. Volume: 4 Issue: 6. ISSN: 2321-8169.

Newhouse, K., Wan, A., Wightman, S. (2014). Lessons from the 2014 Drought: Water Conservation and California Vineyards. Dow Sustainability Fellowship White Paper. University of Michigan School of Natural Resources and Environment.

Radi, Murtiningrum, Ngadisih, Muzdrikah, F. S., Nuha, M.S, Rizqi, F.A.. (2018) Calibration of Capacitive Soil Moisture Sensor (SKU:SEN0193). 4th International Conference on Science and Technology (ICST), Yogyakarta, Indonesia.

Raspberry PI (2020). Raspberry Pi 3 Model B+ https://www.raspberrypi.org/products/raspberry -pi-3-model-b-plus/

Shahzadi, R. et al. (2016). Internet of Things based Expert System for Smart Agriculture. (IJACSA) International Journal of Advanced Computer Science and Applications, Vol. 7, No. 9, 2016. 
Shortt, R., Verhallen, A., \& Fisher, P. (2011). Monitoring Soil Moisture to Improve Irrigation Decisions. FactSheet Ministry of Agriculture, Food and Rural Affairs. Ontario, Canada. http://www.omafra.gov.on.ca/english/engineer/f acts/11-037.htm

Texas Instruments Inc. (2015). LM555 Timer Data Sheet. SNAS548D, Texas Instruments Incorporated.

Vernier Software and Technology. (2017) Vernier LabQuest ${ }^{\mathrm{TM}}$ Reference Guide Version 1.1 .

Zotarelli, L., Dukes, M.D., Morgan, K.T. (2010) Interpretation of Soil Moisture Content to Determine Soil Field Capacity and Avoid OverIrrigating Sandy Soils Using Soil Moisture Sensors. AE460 University of Florida Institute of Food and Agricultural Sciences: Gainesville, FL. 